\title{
Virus Associated Periodontal Diseases: Futuristic Implications
}

\author{
Amit Parashar ${ }^{1 *}$, Sheetal Sanikop ${ }^{1}$, Abhishek Zingade ${ }^{1}$, Shashi Parashar ${ }^{2}$ and Shikha Gupta ${ }^{3}$ \\ ${ }^{1}$ Department of Periodontics, KLE VK Institute of Dental Sciences, Belgaum, Karnataka, India \\ ${ }^{2}$ Division of Virology, Defence R\&D Establishment (DRDE), Jhansi Road, Gwalior, MP, India \\ ${ }^{3}$ Department of Pedodontics, People's Dental Academy, People's University, Bhopal, India
}

Received: August 28, 2014; Accepted: January 06, 2015; Published: January 26, 2015

*Corresponding author: Dr. Amit Parashar, MDS, FAGE, FPFA, Department of Periodontics, KLE VK Institute of Dental Sciences, Belgaum, Karnataka 590010, India, Tel: 9685-480-824; Fax: 831-2470-640 E-mail: captamitparashar@gmail.com

\begin{abstract}
Periodontal diseases have alarmed the current scenario globally. Still very less evidences has been clued in this area. Previously it was thought that virus is not involved with severe cases of periodontitis. In 20th century, virus association of periodontal cases has shown for the first time. After that several studies covered the strong evidences about direct virus isolation from deep focal sites. The articles published recently in journals of repute has shown a very strong evidences in the involvement of human herpes virus, Epstein Barr virus, Human cytomegalovirus, Human Immunodeficiency Virus in both chronic as well aggressive periodontitis. Our main aim of this review is to have compiled information in this article for what have been done in the area and remaining futuristic approach; as a current concern. This will not only help in early diagnostic and virus characterization as well identification of biomarkers for disease. The compiled information and critical aspects in the area will also help to develop suitable diagnostics and therapeutics.
\end{abstract}

Keywords: Virus; Molecular Methods; Culture; Periodontitis

\section{Introduction}

Periodontal diseases affects millions of people worldwide still few of them could get proper treatment due to non availability of actual causing agent for the disease. Oral cavities harbor millions of unidentified viruses [1,2]. As the research work progressed periodontitis is now known as the most complex infectious diseases attribute to multiple viral agents which intern induce host immune responses. The periodontal disease is a chronic and degenerative disease that affects and remains localized on gingiva, periodontal ligament, cementum and alveolar bone. As the presence of viral strains identified in association of periodontal diseases a new turning point was observed in this research area in 21st century [3,4]. But still the progress is very poor in the area according to available update technologies in the century. However, not much detailed data is available for virus involvement and host responses in the pathogenesis of periodontitis. Detection and quantification of periodontopathic viral species are useful for identifying subjects at elevated risk of periodontitis, but do not consistently predict clinical outcome.
These uncertainties have given targeted efforts to find viral etiologic factors for periodontitis.

Individual periodontal lesions harbor millions genomic copies of Human Herpesviruses (HHV), Human Cytomegalovirus (HCMV), Epstein-Barr Virus (EBV), Human Papillomavirus (HPV), Human Immunodeficiency Virus (HIV), Hepatitis B And C Virus (HBV, HCV), and Human Enterovirus (HEV). Out of them EBV and CMV are the most commonly researched virus in periodontology and more than 1 million genome copies identified at a single site of infection (Figure 1)[2]. The host immune response attempts to control pathogenic viruses in periodontal sites. However, it is unclear if various immune mediators, such as certain cytokines and chemokines, exert primarily a protective or a destructive role in periodontal disease. Conventionally viruses are much a challenge task for detection and treatment as compared to bacteria. The main bottleneck in this area is to identify the initial stage of the disease and distinguish it properly from initial and late stage. For causing any infection in any host at its particular target sites the conditions proposed to have: Pathogenicity; that is likelihood of causing any disease, Virulence: ability of microorganism to cause any disease (virulent and non-virulent strain), Virulence factors: gene products that enhance potential to establish infection, attachment, evade host immune response, proliferation, damage to host tissue, and transmission (Figure 2 and 3).

Also the adopted survival strategy of the organism in the host environment and favorable selection of microbial species in the environment known as survival of the fittest is also plays a major role in causing viral infections. That is also known as selection pressure coupled with environmental changes. When the equilibrium of host body disturbs it lead to pathology of disease. Due to lack of proper data about infectious and clinical periodontal breakdown that has given rise number of hypothesis about etiology. Few of them have suggested infectious agent like virus is involved with disease mechanism while other emphasize on host immune factor and genetic characteristics.

Major advances in the diagnosis, prevention and treatment 


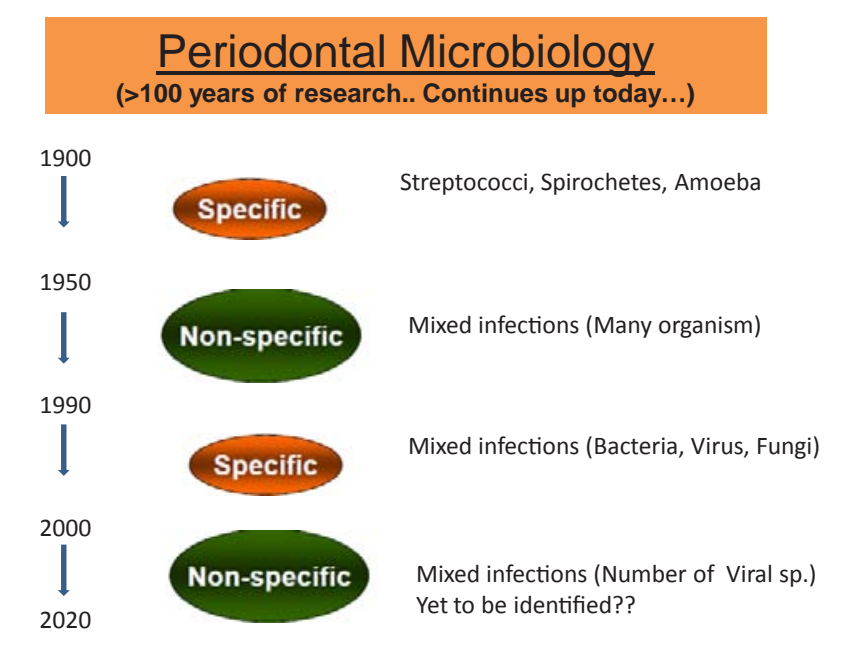

Figure 1: Microorganism associated with periodontal diseases.

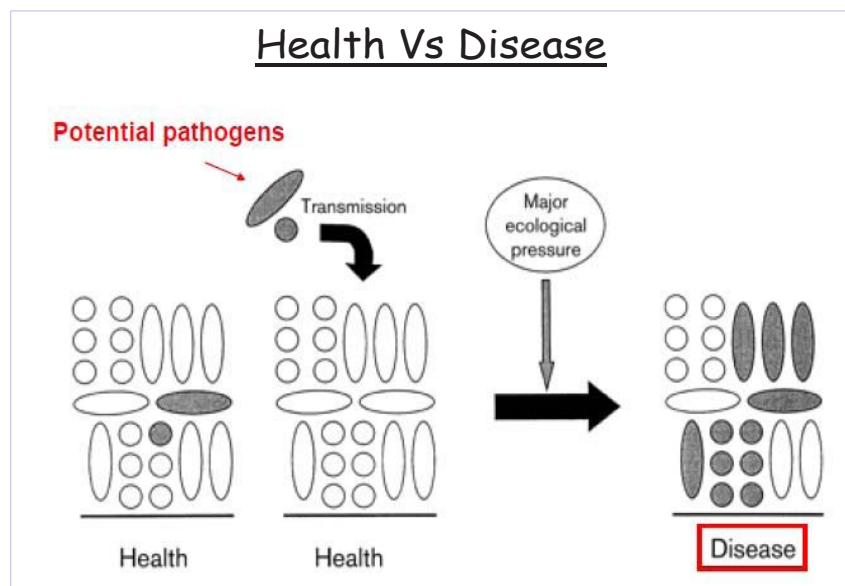

Figure 2: How an organism gets disease after infection.

of periodontitis probably depends upon a better understanding of the pathogenic infections and the associated host responses. The main aim behind this review is to decipher precise role of viruses that may be potentially involved in periodontal disease, and to evaluate the evidence supporting the hypothesis that viral infection plays a role in the development of periodontal disease.

\section{Virus presence in periodontal cases}

In 21st century new insights into the oral microbial etiology deciphered the precise role of viruses involved in progression of periodontal cases. HIV positive patients harbor greater numbers of HHV in periodontal pockets [5]. HCMV infection also causes acute infection in a patient infected with HIV was also deciphered. During childhood, many people acquire primary infection with HCMV [6-8]. In case of reactivation of these viruses very chronic infection may occur. Severe disease caused by HCMV is life-threatening in the HIV infected population [7]. Periodontal occurrences of HHV-6, HHV-7 and HHV-8 have been reported in HIV seropositive and 14 HIV negative adults (Figure 4) $[9,10]$. The human periodontium has been investigated for a site of infection or reservoir for HCMV, EBV-1, EBV-2, HSV, HHV6 and 7. Human papillomavirus (HPV) is also found associated with periodontal cases. HPV-16 is detected in oral mucosa; it was proved through investigation of this virus from periodontal lesions at disease sites [11-14].

This was also studied well that consequences and disease progression risk is much higher in case of virus and bacterial coinfection in periodontitis. Along with direct involvement of viruses in periodontal cases few medically compromised patients may experience repeated and prolonged HHV re-activation, which may be an important reason for the observed advanced types of periodontitis. However a recent fact is that now we are many steps ahead from assessment of microbiota that could be isolated from lab cultures. As a futuristic and an updated research tool next -generation sequencing technology is required to understand the vast depth of oral viral flora. The huge amount of data obtained from this technique will not only help to make out presence of new viral species but also help to utilize present available diagnostic tools against known viral species like HHV, HIV etc. With the advent of Next-Generation Sequencing (NGS) technologies, researchers were now focusing on in-depth study for profiling of the microbiomes and metagenomes. The integration of bacterial, viral, fungal "metaomes" such as the

\section{VIRUS IN PERIODONTITIS}

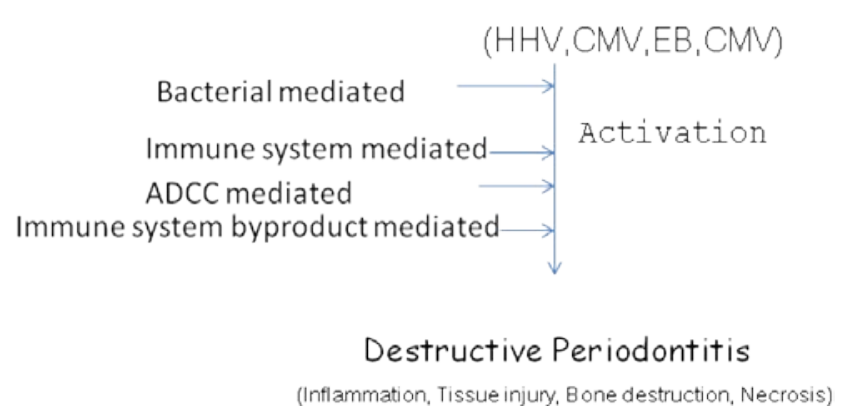

Figure 3: Generalized mechanism for virus induced periodontal diseases.

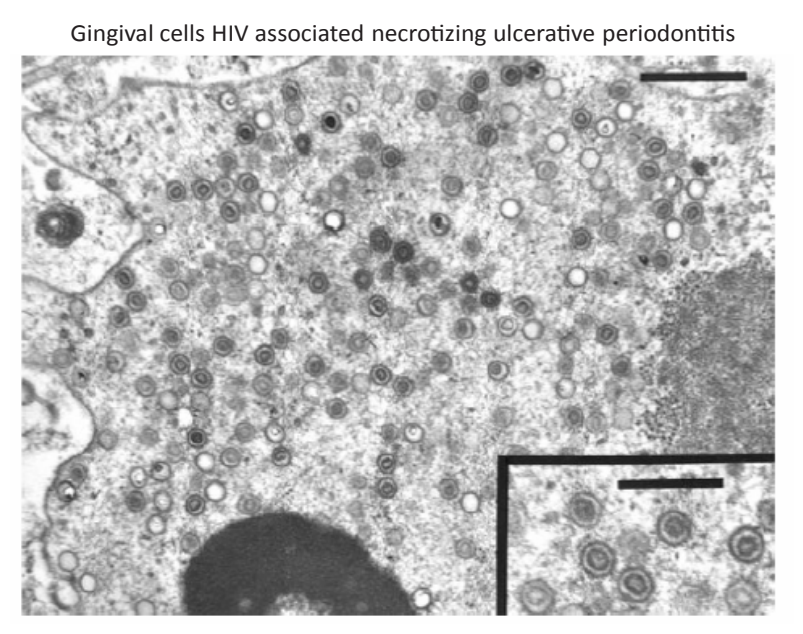

Figure 4: Virus induced necrotic ulcers in periodontal case. 
meta-transcriptome, meta-proteome, and metametabolome, together with the host as a major co-factor, should be the ultimate goal in unraveling the complexity of the oral interactome.

\section{Pathogenesis of virus-associated periodontal disease}

HHV has been studied mainly as periodontal disease pathogenesis model (Figure 5). In the case of HHV it was reported that after having a lytic cycle viral presence remains in latent form throughout the life of host. Reactivation of this virus after a periodic interval may be due to stress or change in host immune status which may lead to encephalitis, pneumonia, hepatitis and various form of cancer is a serious concern $[15,16]$. Periodontal tissue breakdown occurs more frequently and progress more rapidly in HHV infected periodontal sites. HHV may cause periodontal pathology as a direct result of virus infection and replication, or as a result of virally mediated damage to the host defense. HHV may exert periodontopathic potential through at least five mechanisms, operating alone or in combination. HHV may cause direct cytopathic effects on fibroblasts, keratinocytes, endothelial cells on inflammatory cells such as polymorphonuclear leukocytes, lymphocytes, macrophages and possibly on bone cells, HHV cytopathic effects may hamper tissue turnover and repair [17-20].

HHV periodontal infections may impair cells involved in host defense, thereby predisposing to microbial superinfection. HCMV and EBV-1 can infect or alter functions of immune cells mainly monocytes, macrophages and lymphocytes [21,22]. Gingival HHV infection may promote subgingival

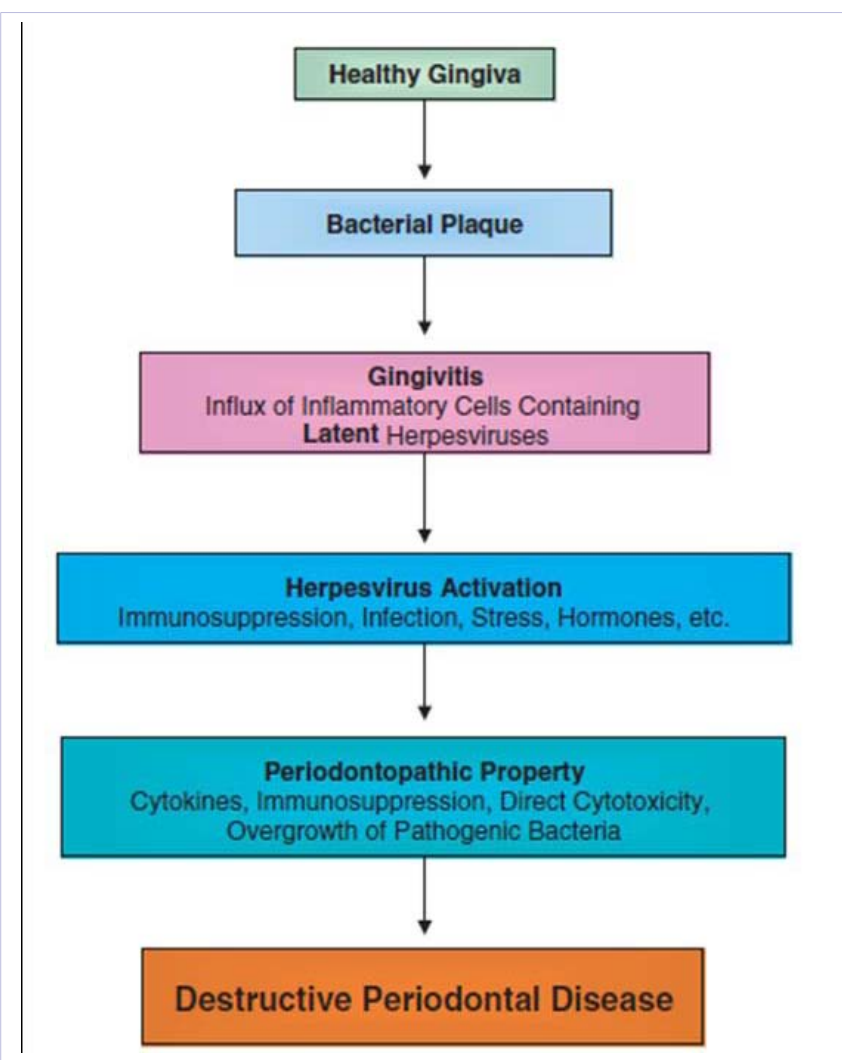

Figure 5: Herpes Virus in destructive periodontal disease (Slots, 2005). attachment and colonization of periodontopathic bacteria, similar to the enhanced bacterial adherence to virus-infected cells. The main reason behind it is viral proteins expressed on eukaryotic cell membranes which act as bacterial receptors and generate new bacterial binding sites. HHV infections give rise to altered inflammatory mediator and cytokine responses [17-20]. Upregulation interleukin 1-beta (IL-1ß) and tumor necrosis factor-alpha (TNF- $\alpha$ ) gene expression of monocytes and macrophages reported in case of HCMV which further lead to enhanced susceptibility to destructive periodontal disease. This may also lead to upregulation of matrix metalloproteinase, downregulation of tissue inhibitors of metalloproteinase and mediate periodontal bone destruction. HCMV and HHV induce cell-mediated immunosuppression by reducing the cell surface expression of MHC (Major Histocompatibility Complex) class I molecules, thereby interfering with T-lymphocyte recognition. In addition, HCMV suppress antigen-specific cytotoxic T-lymphocyte functions, resulting in decreased in circulating CD4+ cells and increased in CD8+ suppressor cells, which in turn may lead to global impairment of cell-mediated immunity [23-26].

\section{Virus diagnosis}

Virus diagnosis is a challenging task in periodontal cases; however the revolution in the field of diagnostic made it easy now a day. Culture of virus in in vitro (cell lines) and in vivo (animal) model was a gold standard but still a time consuming method. Rapid diagnosis of virus is essential by using molecular techniques like PCR, RT-PCR, Real time RT-PCR and sequencing. Periodontal viruses may be identified successfully by using diagnostic DNA microarrays that are able to detect simultaneously $\mathrm{HHV}$, EBV, and CMV. It uses multiplex real-time PCR techniques to quantify simultaneously the number of genome-copies. HHV, HCMV and EBV have been isolated from periodontal disease sites and were confirmed through molecular assays. The presence of HHV also confirmed through DNA probes, Flow cytometry, immunofluorescence staining and culture. The human oral cavity is full of viral species yet to be identified in much detail using various metagenomics approaches [27-32].

\section{Therapeutic implications}

Conventional periodontal therapy can reduce the periodontal load of viruses. Mechanical debridement may be used to suppression of subgingival EBV and CMV.

Anti-HHV chemotherapy can also decrease the salivary viral load. A short course of Valacyclovir, $2 \mathrm{~g}$ twice on the day of treatment and $1 \mathrm{~g}$ twice the following day, resulted in a significant decrease in the salivary occurrence of EBV. Valacyclovir therapy, $3 \mathrm{~g}$ per day for 14 days, resulted in a reduction, of more than 100 fold, of Epstein-Barr virus genome-copies in oral wash fluid of patients with acute infectious mononucleosis. Chemotherapeutics are effective against viruses in the lytic phase, but not against viruses in the latent phase, limiting their potential use to diseaseactive infections. Acyclovir types of drugs are acyclic nucleoside analogues that inhibit HHV replication [33-36]. Future management of periodontal diseases may benefit from anti-viral immunotherapy: either prophylactic vaccines, which harness 
the immune system of healthy subjects to prevent infection with disease-causing viruses; or therapeutic vaccines, which stimulate the immune system into combating existing viruses and disease.

\section{Conclusion}

Better understanding of etiology of periodontitis is critical for developing detection systems and therapies that will enable us to ensure long lasting disease control $[37,38]$. This review article mainly focuses impact of virus associated clinical outcomes in periodontal cases. Present clues of this pathogenic flora of periodontium has a futuristic vast area to research on diagnostic as well as therapeutics. Research during the past 15 years has implied that numbers of viruses are involved in the etiopathogeny of destructive periodontal disease. Published literature shows presence of highly virulent viral species that may lead to severe infections in form of aggressive and chronic periodontitis. Coinfection of two viruses like EBV and CMV is also responsible for progressive periodontitis. HHV also cooperate with specific bacteria in periodontal tissue breakdown. A co-infection of active Herpes viruses and periodontopathic bacteria may constitute a major cause of periodontitis and explain a number of the clinical characteristics of the disease. The ability of an active virus infection to alter the periodontal immune responses may constitute a crucial pathogenetic feature of periodontitis. An active viral infection can exert direct cytopathogenic effects on key cells of the periodontium; induce the release of proinflammatory cytokines [39].

Ongoing research on viral infections of the periodontium has stream lighted towards prevention and treatment of virus induced periodontitis. Studies in the field of virus induced periodontal infections will help in understanding clinical and biological features involved in periodontitis, and also to formulate a new strategies for managing the disease. Detection or quantification of periodontal viruses may prove to have prognostic significance. In order to achieve the final goal to treat any patient up to the maintenance stage so that he/she would be able to get rid of infection truly is at far long. Assessment of the re-activation status of a periodontal infection may help to guide the treatment of patients with severe periodontitis. Development of new vaccines for viruses involved in periodontal disease is required in future which can real hope for low-cost prevention of periodontitis in large groups of individuals. A full understanding of the microbial factors, their pathogenicity as well as host factors are of essential importance for pathogenesis of periodontal disease. In this way it would be possible to treat the periodontal patients adequately.

Therefore to make an effort towards deep understanding regarding etiopathogenesis of disease is required for the development of suitable preventive and therapeutic measures.

\section{References}

1. Slots J. Oral viral infections of adults. Periodontol 2000. 2009; 49:6086. doi: 10.1111/j.1600-0757.2008.00279.x.

2. Cappuyns I, Gugerli P, Mombelli A. Viruses in periodontal disease-a review. Oral Dis. 2005; 11(4): 219-229.

3. Prato G.P, Rotundo R, Magnani C, Ficarra G. Viral etiology of gingival recession. A case report. J Periodontol. 2002; 73(1): 110-114.
4. Maddi A, Scannapieco FA. Oral biofilms, oral and periodontal infections and systemic disease. Am J Dent. 2013; 26(5): 249-54.

5. Jordan L. Periodontal pathogens and reactivation of latent HIV infection: a review of the literature. J Dent Hyg. 2013; 87(2): 59-63.

6. Contreras A, Botero JE, Slots J. Biology and pathogenesis of cytomegalovirus in periodontal disease. Periodontol 2000 2014; 64 (1): 40-56. DOI: 10.1111/j.1600-0757.2012.00448.x.

7. Slots J. Update on Human cytomegalovirus in destructive periodontal disease. Oral Microbiol Immunol. 2004; 19(4): 217-223.

8. Britt WJ, Alford CA. Cytomegalovirus. In: Fields BN, Knipe DM, Howley PM editors. Fields Virology, 3rd edn. Philadelphia: Lippincott-Raven, 1996: 2493-2524.

9. Myrit M, Odden K, Schreurs O, Halstensen TS, Schenck K. The gingival plasma cell infiltrate in HIV-positive patients with periodontitis is disorganized. J Clin Periodontol. 1999; 26(6):358-365.

10. Myrit M, Yuan ZN, Schenck K. Reduced numbers of Langerhans cells and increased HLA-DR expression in Keratinocytes in the oral gingival epithelium of HIV-infected patients with periodontitis. J Clin Periodontol. 2000; 27(7): 513-519.

11. Miller CS, Berger JR, Mootoor Y, Avdiushko SA, Zhu H, Kryscio RJ. High prevalence of multiple human herpesviruses in saliva from human immunodeficiency virusinfected persons in the era of highly active antiretroviral therapy. J Clin Microbiol. 2006; 44(7):2409-2415.

12. Slots J, Sugar C, Kamma JJ. Cytomegalovirus periodontal presence is associated with subgingival dialister pneumosintes and alveolar bone loss. Oral Microbiol Immunol. 2002; 17(6): 369-374.

13. Jiang M, Abend JR, Johnson SF, Imperiale MJ. The role of polyomaviruses in human disease. Virology. 2009; 384(2): 266-273. doi: 10.1016/j. virol.2008.09.027.

14. Nair S, Pillai MR. Human papillomaviruses and disease mechanisms: relevant to oral and cervical cancers. Oral Dis. 2005; 11(6):350-359.

15. Warinner C, Rodrigues JF, Vyas R, Trachsel C, Shved N, Grossmann J, et al. Pathogen and host immunity in the ancient human oral cavity. Nat Genet. 2014; 46(4):336-344. doi: 10.1038/ng.2906.

16. Ly M, Abeles SR, Boehm TK, Robles-Sakisaka R, Naidu M, SantiagoRodriguez T, et al. Altered oral viral ecology in association with periodontal disease. M Bio 2014; 5(3):e01133-14. doi: 10.1128/ mBio.01133-14.

17. Passariello C, Palamara A, Gracie E, Pasquantonio G. Herpesviruses and periodontal disease: a cautionary tale. Int J Immunopathol Pharmacol. 2009; 22(2):263-268.

18. Al-Otaibi LM, Al-Sulaiman MH, Teo CG, Porter SR. Extensive oral shedding of human herpesvirus 8 in a renal allograft recipient. Oral Microbiol Immunol. 2009; 24(2):109-115. doi: 10.1111/j.1399302X.2008.00481.X.

19.Slots J. Herpesviruses, the missing link between gingivitis and periodontitis? J Int Acad Periodontol. 2004; 6(4):113-119.

20. Hochman N, Rones Y, Ehrlich J, Levy R, Zakay-Rones Z. Antibodies to herpes simplex virus in human gingival fluid. J Periodontol. 1981; 52(6):324-327.

21. Sabeti M, Daneshmand A, Simon JH, Slots J. Cytomegalovirus infected inflammatory cells in dental periapical lesions. Oral Microbiol Immunol. 2009; 24(5):434-436. doi: 10.1111/j.1399-302X.2009.00540.x.

22. Vincent-Bugnas S, Vitale S, Mouline CC, Khaali W, Charbit Y, Mahler $\mathrm{P}$, et al. EBV infection is common in gingival epithelial cells of the 
periodontium and worsen during chronic periodontitis. PLoS One 2013; 8 (12):e80336. doi: 10.1371/journal.pone.0080336.

23. Sunde PT, Olsen I, Enersen M, Beiske K, Grind B. Human cytomegalovirus and Epstein-Barr virus in apical and marginalperiodontitis: a role in pathology? J Med Virol. 2008; 80(6):1007-1011. doi: 10.1002/ jmv.21180.

24. Slots J, Saygun I, Sabeti M, Kukar A. Epstein-Barr virus in oral diseases. J Periodontal Res. 2006; 41(4):235-244.

25. Grind B, Olsen I. Do cytomegalovirus or Epstein-Barr virus play a role in periodontitis? In: Gluckman TR, ed. Herpesviridae: viral structure, life cycle and infections. New York: Nova Science; 2009. p. 167-78.

26. Walling DM, Brown AL, Etienne W, Keitel WA, Ling PD. Multiple Epstein-Barr virus infections in healthy individuals. J Virol 2003: 77: 6546-6550. doi: 10.1128/JVI.77.11.6546-6550.2003.

27. Konstantinidis A, Sakellari D, Papa A, Antoniadis A. Real-time polymerase chain reaction quantification of Epstein--Barr virus in chronic periodontitis patients. J Periodontal Res. 2005; 40(4):294298.

28. Kubar A, Saygun I, Yapar M, Ozdemir A, Slots J. Real-time PCR quantification of cytomegalovirus in aggressive periodontitis lesions using TaqMan technology. J Periodontal Res. 2004; 39(2):81-86.

29. Koyama K, Uobe K, Tanaka A. Highly sensitive detection of HPV-DNA in paraffin sections of human oral carcinomas. J Oral Pathol Med. 2007; 36(1):18-24.

30. Luo C-W, Roan C-H, Liu C-J. Human papillomaviruses in oral squamous cell carcinoma and pre-cancerous lesions detected by PCR-based gene-chip array. Int J Oral Maxillofac Surg. 2007; 36(2):153-158.

31. Engelmann I, Petzold DR, Kosinska A, Hepkema BG, Schulz TF, Heim A, et al. Rapid quantitative PCR assays for the simultaneous detection of herpes simplex virus, varicella zoster virus, cytomegalovirus, EpsteinBarr virus, and human herpesvirus 6 DNA in blood and other clinical specimens. J Med Virol. 2008; 80(3):467-77. doi: 10.1002/jmv.21095.

32. Saygun I, Kubar A, Sahin S, Sener K, Slots J. Quantitative analysis of association between herpesviruses and bacterial pathogens in periodontitis. J Periodontal Res. 2008; 43(3):352-359.

33. Sunde PT, Olsen I, Enersen M, Grinde B. Patient with severe periodontitis and subgingival Epstein-Barr virus treated with antiviral therapy. J Clin Virol. 2008; 42(2):176-178. doi: 10.1016/j. jcv.2008.01.007.

34. Miller CS, Avdyushko SA, Kryscio RJ, Danaher RJ, Jacob RJ. Effect of prophylactic valacyclovir on the presence of human herpesvirus DNA in saliva of healthy individuals after dental treatment. J Clin Microbiol. 2005; 43(5):2173-2180.

35. Balfour HH Jr, Hokanson KM, Schacherer RM, Fietzer CM, Schmeling DO, Holman CJ, et al. A virologic pilot study of valacyclovir in infectious mononucleosis. J Clin Virol. 2007; 39(1):16-21.

36. Patel R. Valacyclovir: development, clinical utility and potential. Expert Opin Investig Drugs. 1997; 6(2):173-189.

37. Ezzo PJ, Cutler CW. Microorganisms as risk indicators for periodontal disease. Periodontol 2000. 2003; 32:24-35.

38. Slots J, Rams TE. New views on periodontal microbiota in special patients categories. J Clin Periodontol. 1991; 18(6):411-420.

39. Lin SS, Chou MY, Ho CC, Kao CT, Tsai CH, Wang L, et al. Study of the viral infections and cytokines associated with recurrent aphthous ulceration. Microbes Infect 2005; 7(4):635-644. 